\title{
A novel data-driven rollover risk assessment for articulated steering vehicles using RNN
}

\author{
Keywords: \\ Articulated steering vehicles \\ - Lateral stability \\ - Data-driven \\ - Recurrent neural network \\ - Rollover risk \\ Correspondence to: \\ Qingyuan Zhu \\ zhuqy@xmu.edu.cn
}

\section{Citation:}

Chen, X., Chen, W., Hou, L., Hu, H., Bu, X., Zhu, Q. (2020). A novel data-driven rollover risk assessment for articulated steering vehicles using RNN. Journal of Mechanical Science and Technology 34 (5) (2020) 2161 2170

http://doi.org/10.1007/s12206-020-0437-4

\author{
Xuanwei Chen ${ }^{1}$, Wei Chen ${ }^{1}$, Liang Hou', Huosheng $\mathrm{Hu}^{2}$, Xiangjian Bu${ }^{1}$ and Qingyuan $\mathrm{Zhu}^{1,3}$ \\ ${ }^{1}$ Department of Mechanical and Electrical Engineering, Xiamen University, Xiamen 361005, China, \\ ${ }^{2}$ School of Computer Science \& Electronic Engineering, University of Essex, Colchester CO4 3SQ, UK, \\ ${ }^{3}$ Fujian Collaboration Innovation Centre for R\&D of Coach and Special Vehicle, Xiamen University of \\ Technology, Xiamen 361005, China
}

\begin{abstract}
Articulated steering vehicles have outstanding capability operating but suffer from frequent rollover accidents due to their complicated structure. It is necessary to accurately detect their rollover risk for drivers to take action in time. Their variable structure and the variable center of mass exhibit nonlinear time-variant behavior and increase the difficulty of dynamic modelling and lateral stability description. This paper proposes a novel data-driven modelling methodology for lateral stability description of articulated steering vehicles. The running data is first collected based on the typical operations that prone to rollover and then classified into two types: Safety and danger. The data quality is further improved by wavelet transformation. Finally, an RNN model is built on the data. The experimental results show that the output of the RNN model can accurately quantify lateral stability of the vehicle, i.e., the risk of rollover, when it is turning and crossing uneven surfaces or obstacles.
\end{abstract}

\section{Introduction}

Articulated steering vehicles have two separate bodies connected by a steering hinge, which reduces the turning radius and enhances the load-bearing capacity. This mechanism also gives the vehicle enough room to install tires with large diameter and wide tread to save its cost and improve its trafficability. With these features, the articulated steering vehicles (e.g., articulated wheel loaders, scrapers and articulated trucks) are widely used in agriculture and construction industries. However, they are prone to rollover accidents due to their articulated steering mechanism and high center of gravity [1].

To prevent rollover accidents, it is necessary to design a successful active safety warning system to notice any potential rollover danger. In other words, the meaningful and accurate rollover risk assessment is an important step in developing rollover avoidance schemes. Some existing studies addressed the risk assessment issue of front wheel steering vehicles [2-4], in which a dynamics model of front wheel steering vehicles was first built and the lateral stability of the vehicles was then quantitatively described for the rollover risk assessment. The same method has been adopted in the articulated steering vehicle.

Due to the articulated mechanism, an articulated steering vehicle has higher degrees-offreedom (DOF) and requires higher dimensional modelling to cover the dynamic characteristics. Li et al. [5] built a 7 degrees-of-freedom (DOF) nonlinear dynamics model and Chi et al. [6] even built a 12-DOF model. These models describe the dynamic characteristics of articulated steering vehicles well and are only suitable for theoretical research, as their calculation process is relatively complicated and is not suitable for real-time calculations.

On the other hand, some simplified models were built to explore the horizontal movement [7], steering instability [8] and snaking behavior [9] of articulated steering vehicles. These simplified models do not require complicated calculations. However, they can only characterize the specific working condition of an articulated steering vehicle and need to be adjusted for different 


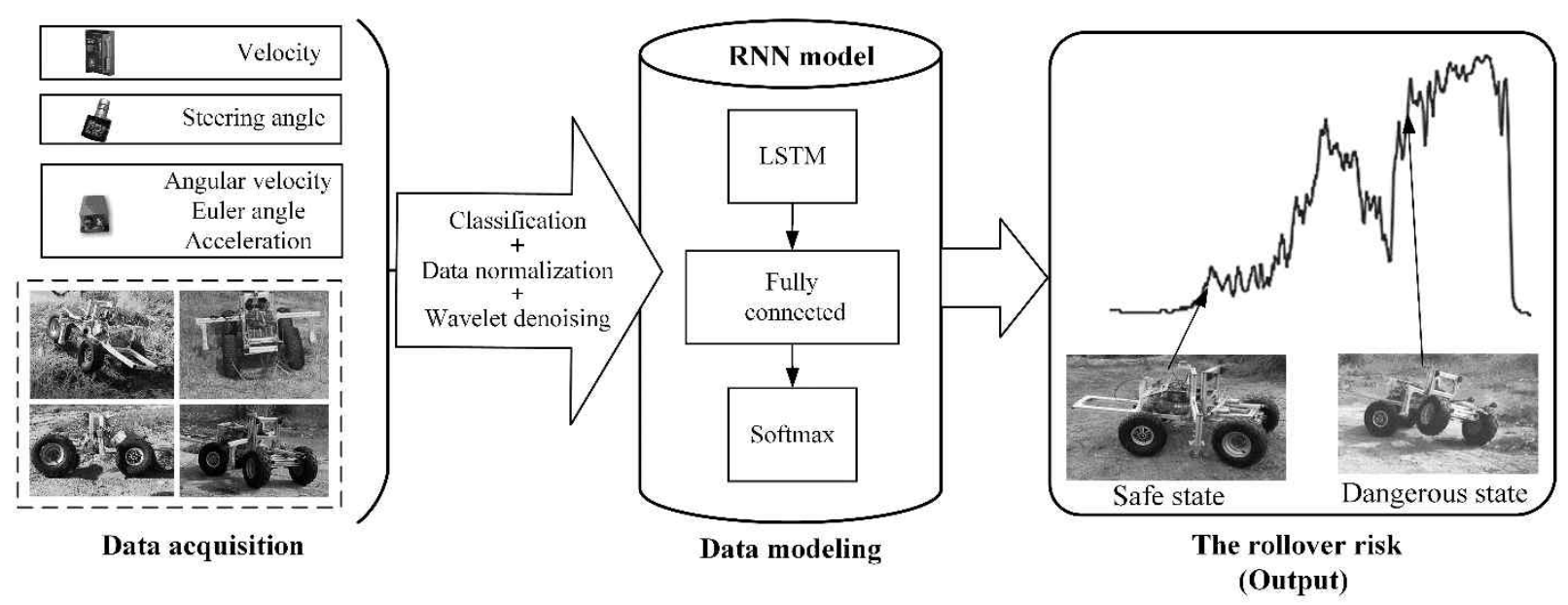

Fig. 1. A novel data-driven rollover risk assessment framework for articulated steering vehicles.

working conditions. Furthermore, the risk assessment indicators proposed based on these models are limited in describing the static risk in the special condition because they cannot explore the trend of critical data during the rollover comprehensively.

Recently, data-driven modelling methods have shown numerous achievements in nonlinear systems [10-12] because of their universal function approximation properties. A model was built based on representative data using machine learning algorithms to approach the real situation. They do not require complex higher-order equations to describe complex nonlinear system characteristics and therefore reduce the cost and time. In the vehicle research, the method is used to classify the lateral stability of heavy trucks [13], analyze vehicle driving behavior $[14,15]$ and build a path tracking model for autonomous vehicles [16, 17], and all these researches show promising results. Hence, unlike previous works on dynamic modelling, we could develop a data-driven model to assess rollover risk based on vehicle running data that all are the crucial data affecting vehicle lateral stability and can be acquired from diverse sensors.

Rollover is a dynamic process and its historical data provides important references for whether the vehicle will have a potential rollover soon. To explore the trend of critical data during the rollover, we chose recurrent neural network (RNN) modelling architecture, which is able to map from the entire history of previous inputs to target vectors and allow a memory of previous inputs to be kept in the network's internal state [18]. It works well on the modelling problem that the inputs at different times are associated with each other, such as time series analysis [19], natural language processing [20] and robot path planning [21]. Compared to other algorithms that can only map from input data to target vectors, it has the advantage of dealing with the modelling problem of rollover risk assessment.

In this paper, we used RNN to build a data-driven rollover risk assessment model based on vehicle running data. First, we controlled the proportional prototype to do the most prone rollover operation for data acquisition. Secondly, we normalized and denoised the collected data and divided it into two categories. Thirdly, we trained an RNN model to assess the rollover risk of articulated steering vehicle based on the processed data. The experimental results show that the training data based RNN model had the best performance after wavelet transformation. By combining with SoftMax function, the model output can accurately quantify the lateral stability of a vehicle during its turning and crossing over an obstacle.

The rest of the paper is organized as follows. Sec. 2 introduces the method for building the data-driven model, including hardware platform construction, data collection, data processing and RNN model structure. In Sec. 3, experiments are conducted on a home-built wheel loader to demonstrate the performance of model training and the accuracy of the proposed method on rollover risk assessment. Finally, a brief conclusion and future work are presented in Sec. 4.

\section{Methodology}

Fig. 1 shows the proposed framework for data-driven rollover risk assessment articulated steering vehicles. A prerequisite for data-driven modelling is to build a training dataset. Then mathematical mapping expressions are built from the inputoutput relationships of the training dataset. In our previous research, we found velocity, steering angle and attitude are the key signals to reflect the lateral stability of the vehicle, as well as the signals that need to be acquired in the dynamic verification experiment [22]. Velocity is the most important factor in driving safety and one of the indispensable signals to analyze whether the vehicle has a rollover risk. When a vehicle is turning, its steering angle increases and its front and rear bodies rotate, causing the vehicle's center of gravity to shift laterally. An inertial navigation system (INS) can supply the attitude signals, including angular velocity, acceleration and Euler angle $[23,24]$. Before a vehicle has a rollover, the lateral acceleration will change suddenly, and the other attitude signals maybe 


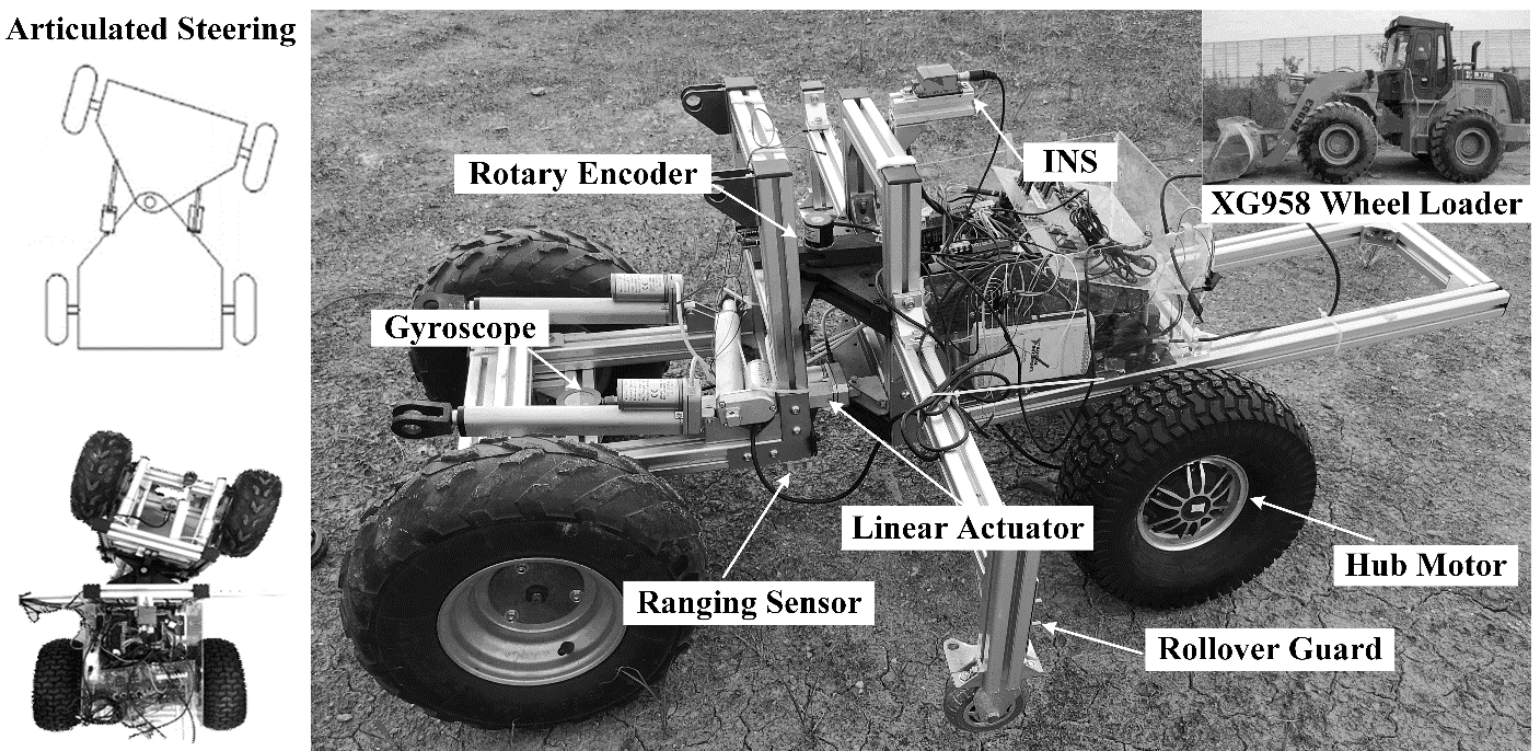

Fig. 2. The home-built scale-down prototype of articulated steering vehicle.

exceed normal. Therefore, we chose these signals as inputs. First, our home-built prototype vehicle is operating under the most prone rollover condition for data acquisition. Then, the collected data are divided into two categories: Safe and dangerous states. In the modelling stage, the processed data are used to improve the data quality which determines the model accuracy. After data processing, an RNN model is built to predict the risk of rollover. The proposed approach in this paper is described in detail in the following sections.

\subsection{Hardware platform construction}

The rollover experiments were difficult to conduct and strongly destructive, which may cause a vehicle crash. In Refs. $[5,10,25]$, the prototype vehicles were used to do verification experiments instead of real articulated steering vehicles. Therefore, we used a home-built articulated steering vehicle (about 1:4 in size to a real XG958 wheel loader made by XGMA, Inc.) for data acquisition and experiments.

As shown in Fig. 2, our prototype vehicle has two separate bodies connected by a steering hinge. The relative rotation of front and rear bodies is realized by a linear actuator mounted at the hinge driving, which is a high-power hub motor controlled by a high resolution remote controller. It has the basic features of a real articulated steering vehicle (i.e., an articulated steering mechanism and a swing rear driving axle), as well as the same dynamic characteristics [22]. Therefore, the prototype vehicle has enough power to drive in various terrains and can be used to collect the critical data during the rollover experiments.

A rollover guard unit is installed on the sides of the prototype, which limits the roll angle of the prototype vehicle to prevent damage from severe rollover accidents. The sensing system consists of a motor driver, a rotary encoder, an INS, a gyroscope and two ranging sensors for measuring velocity, steering angle, attitude and distance from chassis to ground. Table 1 shows the detailed sensor information. These sensors are connected to the data acquisition card (NI USB-6211) for data acquisition and it can accurately collect the data at $100 \mathrm{~Hz}$.

\subsection{Data acquisition}

To acquire the data, we controlled the prototype vehicle to operate under the potential rollover conditions [26]. Fig. 3 shows a sketch map of typical rollover conditions. More specifically, Fig. 3(a) shows that the prototype vehicle has a rollover potential when it is crossing a large obstacle. Fig. 3(b) shows that the vehicle has a rollover potential when it does a fast turn. Fig. 3(c) shows that the vehicle has a potential rollover when it is avoiding one obstacle without slowing down.

In our previous study [27], we found that the general rollover process of an articulated steering vehicle is that the vehicle body first flips along the central axis to the rear body limit block and resists the rear driving axle, then the body drives the rear driving axle to flip together, making the wheels on the same side leave the ground and causing a rollover accident. According to this process, we classify each group of data into safe and dangerous states.

As shown in Fig. 4, the safe state means that the four wheels of the vehicle are on the ground and the vehicle can run stably. A dangerous state means that the vehicle has a large inclination and one wheel leaves the ground. To protect the prototype from damage, the rollover guard unit touches the ground and limits the roll degree of the vehicle. If the lateral force is large enough, the vehicle will roll along the central axis to make the two wheels on the same side leave the ground with the rollover guard, causing a rollover accident.

We used a camera and ranging sensors mounted on the chassis to distinguish the vehicle states and classify each group of data into two states: Safe and dangerous. As the sampling rate of the camera was not high enough, we could 
Table 1. Information of main sensors.

\begin{tabular}{c|c}
\hline Equipment & Specifications \\
\hline INS (Xsens MTi-700) & $\begin{array}{c}\text { Latency: }<2 \mathrm{~ms}, \text { gyroscopes: } 450 \% \text {, acceler- } \\
\text { ometers: } 200 \mathrm{~m} / \mathrm{s}^{2}, 57 \times 42 \times 23 \mathrm{~mm}, 0.055 \mathrm{~kg}\end{array}$ \\
\hline Motor driver & $\begin{array}{c}\text { Output the corresponding pulse frequency ac- } \\
\text { cording to the motor speed, maximum speed: } \\
\text { (Ican Automation } \\
\text { BLD750) }\end{array}$ \\
\hline Rotary encoder & $\begin{array}{c}\text { Incremental, resolution: } 1000 \mathrm{P} / \mathrm{R}, \text { maximum } \\
\text { speed: } 6000 \mathrm{rpm}, \Phi-40 \mathrm{~mm}\end{array}$ \\
\hline
\end{tabular}

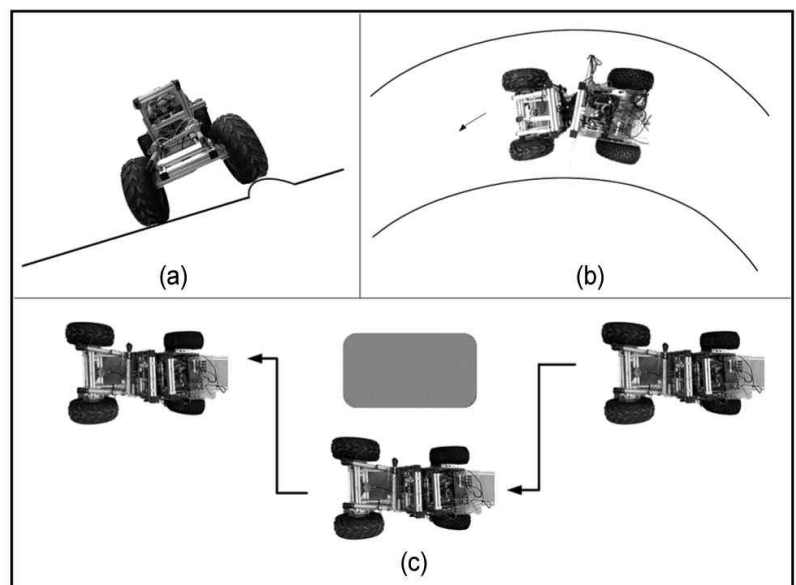

Fig. 3. A sketch map of typical rollover operations: (a) Obstacle crossing (b) turning; (c) obstacle avoidance.

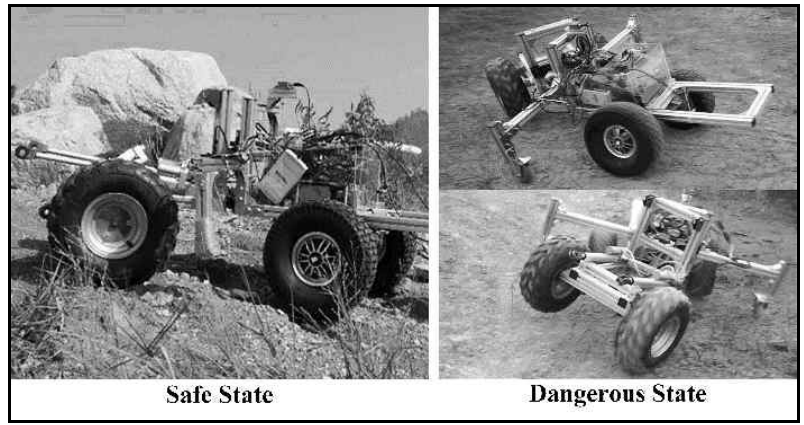

Fig. 4. Safe state and dangerous state of our prototype vehicle.

only determine the scope of the dangerous data roughly by the camera. Then we classified data by using the ranging sensors with the same sampling rat as other sensors in the data acquisition system. Although the distance from two sides of the prototype vehicle to ground acquired from the ranging sensors will be different when the vehicle has a rollover accident, rugged terrain will make the ranging sensors obtain the same result. Therefore, the camera is complementary to the ranging sensors and can achieve accurate data labelling.

\subsection{Data processing}

\subsubsection{Under sampling}

There are 5850 positive samples (vehicle dangerous state)

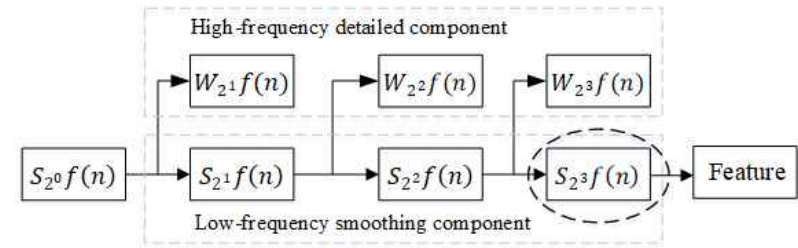

Fig. 5. Process of lateral acceleration signal decomposition.

and 30650 negative samples (vehicle safe state) in the collected data because the positive samples can only acquire in dangerous state which occurs during a short period. To reduce the impact of data imbalance, we used the under sampling method that aims to balance class distribution through extracting the negative samples at equal intervals (i.e., reduce the sampling frequency of negative samples through extraction the raw data at four intervals). After that, the number of negative samples is 7662 , which is equivalent to the number of positive samples.

\subsubsection{Wavelet transformation}

The root cause of vehicle rollover is the sudden change of lateral force, which makes the vehicle unable to maintain a stable state. The lateral acceleration is the signal that reveals most of the changes in the lateral force of the vehicle. However, articulated steering vehicles often travel on rugged terrain and their vehicle bodies are prone to vibrate irregularly, resulting in the collected lateral acceleration containing a large amount of transient signal and noise, thus flooding the useful signal.

Therefore, for lateral acceleration signals, we used the Mallet algorithm [28] to do third-order discrete wavelet transformation, which has shown success in numerous applications from control of automated guided vehicle (AGV) to error compensation on sensors [29, 30]. By applying this method, we can filter out the noise signal caused by the vehicle body's irregular vibration and extract the signal that is most favorable for lateral stability analysis. The formula of the Mallet algorithm is shown below:

$$
\begin{aligned}
& S_{2^{j}} f(n)=\sum_{k} h_{k} S_{2^{j-1}} f\left(n-2^{j-1} k\right) \\
& W_{2^{j}} f(n)=\sum_{k} g_{k} S_{2^{j-1}} f\left(n-2^{j-1} k\right)
\end{aligned}
$$

where $S_{2^{0}} f$ is the input signal, $\mathrm{j}$ is the decomposition scale, $h_{k}$ and $g_{k}$ are the coefficients of the low-pass and high-pass filters, $S_{2^{j}} f(n)$ is the low-frequency smoothing component under the $j_{t h}$ scale, and $W_{2^{j}} f(n)$ is the high-frequency detailed component under the $j_{t h}$ scale.

As shown in Fig. 5, the original signal $S_{2^{0}} f$ is decomposed into the low-frequency smoothing component and the highfrequency detailed component, and then the low-frequency smoothing component is used as the original signal of the next decomposition. The decomposition is repeated three times and the final low-frequency smoothing component is the useful feature for our model. The decomposition is repeated three times and the final low-frequency smoothing component is the 


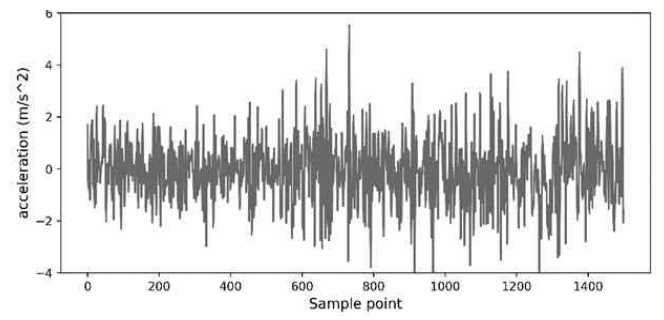

Fig. 6. The raw signal of lateral acceleration.

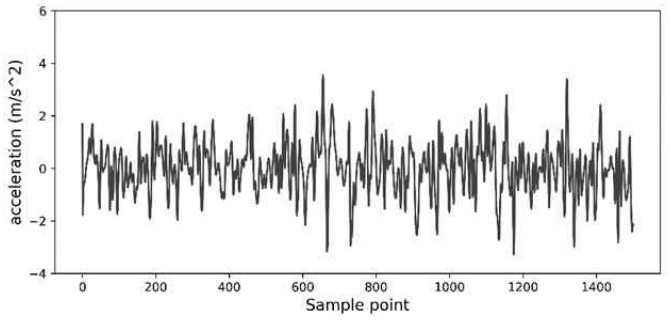

Fig. 7. The signal after wavelet transformation.

useful feature for our model. Fig. 6 shows the raw signal and Fig. 7 shows the lateral acceleration signal after wavelet transformation. The signals after wavelet transformation are smoother than the raw signals.

\subsubsection{Data normalization}

The collected data has different dimensions. For example, the unit of velocity is $\mathrm{km} / \mathrm{h}$ and the unit of lateral acceleration is $\mathrm{m} / \mathrm{s}^{2}$. The difference between the values is very large. The accuracy of the model will be influenced without data normalization.

Z-score is a common normalization method which uses the difference between the original data and its mean value divided by the standard deviation to replace the original data. It reflects the relative standard distance between the data and the mean value, eliminating the effects of different dimensions and guaranteeing comparability between the data. The calculation formula is as follows:

$$
x^{\prime}=\frac{x-\bar{x}}{\sigma}
$$

where $\mathrm{x}$ is the original data; $\bar{x}$ is the mean value of the original data and $\sigma$ is the standard deviation of the original data.

\subsection{Data modelling with RNN}

The historical data can predict whether the vehicle will have a rollover soon. For instance, when the vehicle is in a dangerous state at the last moment caused by high speed or obstacle crossing, the rollover risk will be greater than when it is in a safe state. Another case in point is when the vehicle is driving on the slope at the last moment, the vehicle roll angle is greater than zero; the rollover risk will be greater than when the roll angle is zero. Therefore, we train an RNN model to assess the

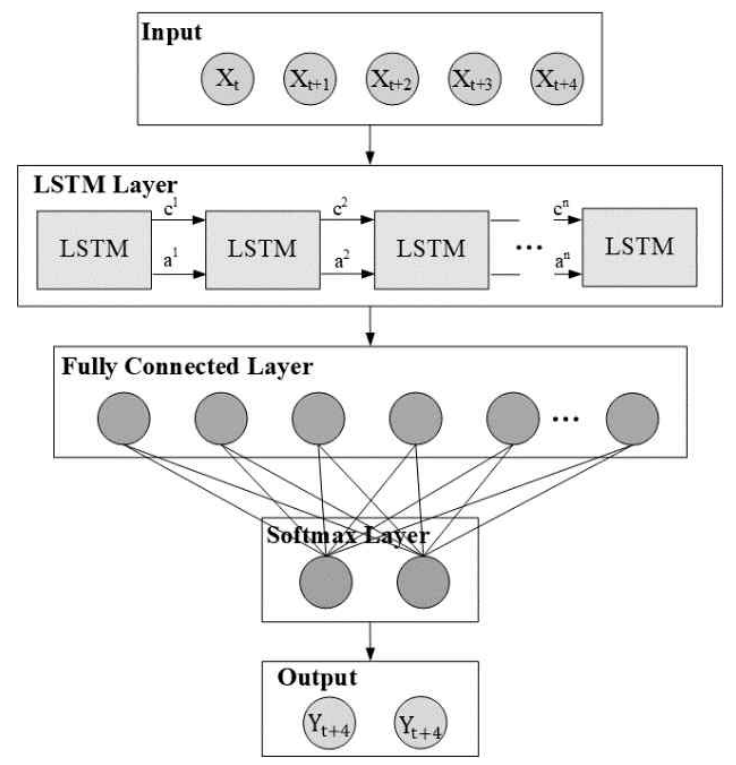

Fig. 8. The network structure of RNN evaluation model.

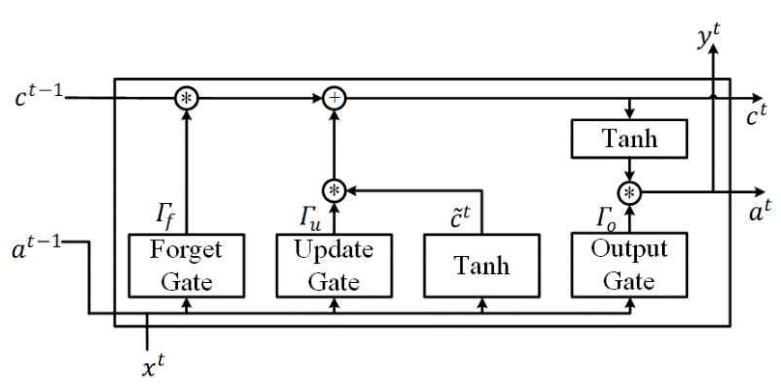

Fig. 9. The LSTM structure.

risk, which allows a memory of previous inputs to be kept in the network's internal state.

The developed RNN model is $y_{t+1}=F\left(y_{t}, y_{t+1}, \ldots, y_{t+3}, x t\right.$, $\left.\mathrm{x}_{\mathrm{t}+1}, \ldots, \mathrm{x}_{\mathrm{t}+4}\right)$ with the input is $\mathrm{X}=\left(\mathrm{x}_{\mathrm{t}}, \mathrm{x}_{\mathrm{t}+1}, \mathrm{x}_{\mathrm{t}+2}, \mathrm{x}_{\mathrm{t}+3}, \mathrm{x}_{\mathrm{t}+4}\right)$. More specifically, its input is the first five moments of data, including velocity, steering angle and attitude, and its output is the rollover risk of the final moment. Fig. 8 shows the network structure that consists of an LSTM layer, a fully connected layer and a SoftMax layer.

LSTM layer: The emergence of LSTM solves the problem of the traditional neural network gradient disappearance and explosion [31]. As shown in Fig. 9, the LSTM unit is composed of a forget gate, an update gate and an output gate. The gate structure makes the LSTM unit learn what will be left and discarded during the training process. The units are connected by $c^{t}$ and $a^{t}$, the formulas are as follows:

$$
\begin{aligned}
& \tilde{c}^{\langle t\rangle}=\tanh \left(W_{c}\left[a^{\langle t-1\rangle}, x^{\langle t\rangle}\right]+b_{c}\right) \\
& \Gamma_{u}=\sigma\left(W_{u}\left[a^{\langle t-1\rangle}, x^{\langle t\rangle}\right]+b_{u}\right) \\
& \Gamma_{f}=\sigma\left(W_{f}\left[a^{\langle t-1\rangle}, x^{\langle t\rangle}\right]+b_{f}\right)
\end{aligned}
$$




$$
\begin{aligned}
& \Gamma_{o}=\sigma\left(W_{o}\left[a^{\langle t-1\rangle}, x^{\langle t\rangle}\right]+b_{o}\right) \\
& c^{\langle t\rangle}=\Gamma_{u} * \tilde{c}^{\langle t\rangle}+\Gamma_{f} * c^{\langle t-1\rangle} \\
& y^{t}=a^{\langle t\rangle}=\Gamma_{o}{ }^{\langle t\rangle}
\end{aligned}
$$

Fully connected layer: The units in the fully connected layer are the same as the common neural network. The effect of the fully connected layer is to increase the nonlinearity of the model, to learn the nonlinear relationship between the data processed by the LSTM layer and the output data.

SoftMax layer: To make the output of the rollover evaluation model not a monotonous category, we introduce the SoftMax function in the last layer of the model, which maps the output to the value of the interval $(0,1)$. Assume that the original output of the neural network is $y_{1}, y_{2}, \ldots \ldots, y_{n}$, then the output processed by the SoftMax function is:

$$
y_{i}^{\prime}=\frac{e^{y_{i}}}{\sum_{j=1}^{n} e^{y_{j}}}
$$

We chose cross entropy as the loss function for back propagation, which is widely used in classification problems and can reflect the similarity between the probability of the model output and the real probability of the sample. $P$ is the true output and $q$ is the model output, and the cross entropy $H(p, q)$ is:

$$
H(p, q)=-\sum_{x} p(x) \log q(x)
$$

\section{Experiments and results}

\subsection{Model evaluation}

The total data for training is 13512 groups, including 5850 positive samples and 7662 negative samples. Each group means one hertz acquisition data. We divide the data into training and test sets randomly, where the training set accounts for $80 \%$ of the total data and the test set accounts for $20 \%$. The RNN model is built on tensorflow1.9.0 and uses the stochastic gradient descent algorithm for back propagation. The training parameters are shown in Table 2.

The RNN model is trained by the raw and processed data, respectively. Fig. 10 shows the change of loss function. The values of the loss function decrease rapidly at the initial stage of training. As the number of iterations increases, the values of the loss function decrease slowly and converge to 0.223 and 0.130 , respectively. The result indicates that the model performance trained by the processed data is better than that trained by the raw data.

Based on the same data, the RNN model in this paper was compared with SVC and GBDT [32]. After debugging, we chose the poly as the kernel function of SVC and made the depth of GBDT 10. We evaluated the model with accuracy, precision and recall. Accuracy refers to the proportion of the number of samples that correctly identify the state of the vehi-
Table 2. The training parameters of RNN.

\begin{tabular}{c|c|c|c|c}
\hline Epoch & Neurons number & Batch size & Learning rate & Activation function \\
\hline 2000 & 50 & 100 & 0.01 & Tanh \\
\hline
\end{tabular}

Table 3. Evaluation results of different models.

\begin{tabular}{c|c|c|c|c}
\hline Data & Method & Accuracy (\%) & Precision (\%) & Recall (\%) \\
\hline \multirow{4}{*}{ Processed } & RNN & 98.3 & 97.8 & 98.0 \\
\cline { 2 - 5 } & SVC & 97.2 & 96.7 & 95.3 \\
\cline { 2 - 5 } & GBDT & 97.6 & 97.2 & 96.2 \\
\hline \multirow{4}{*}{ Raw } & RNN & 95.2 & 96.5 & 88.5 \\
\cline { 2 - 5 } & SVC & 93.5 & 95.3 & 83.9 \\
\cline { 2 - 5 } & GBDT & 94.8 & 96.1 & 87.7 \\
\hline
\end{tabular}

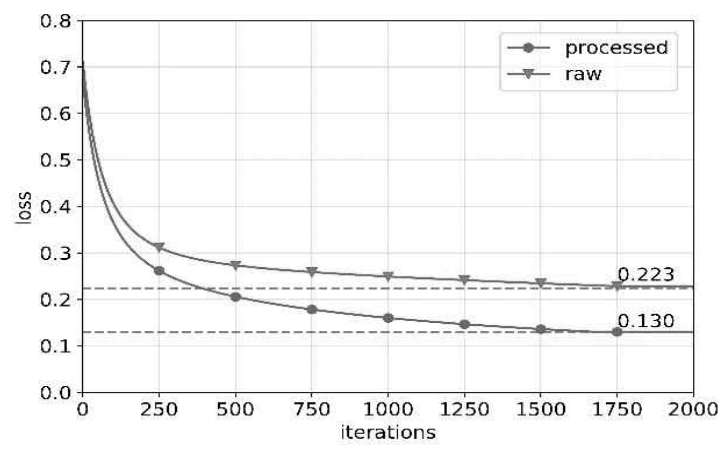

Fig. 10. The change in the value of loss function.

cle to the total number of samples. Precision refers to the proportion of positive samples correctly identified by the model to all positive samples judged by the model. Recall means the proportion of the vehicle dangerous state sample that is correctly identified by the model to all positive samples.

Table 3 presents the evaluation results. The three evaluation indicators of the model trained based on the processed data are better than the model trained based on the raw data, especially the recall rate, which refers to the correct rate of model identification of dangerous state samples. It indicates that the processed data features are helpful for the model to learn the dangerous state. On the other hand, the RNN based three evaluation indicators are better than GBDT and SVC, which proves that RNN is more suitable for our data.

\subsection{Rollover risk assessment}

The details of the risk assessment for articulated steering vehicles are given in Fig. 11. The processed input data is fed into the RNN model to obtain the output. The model output is the probability that the vehicle is either in a safe state or a dangerous state. We only take the probability of a dangerous state to characterize the state of the vehicle at the next moment since the output probabilities are complementary. When the output is less than 0.5 , the vehicle is considered to be in a safe state, and when the output is bigger than 0.5 , the vehicle is in a dangerous state. An increase in output probability means that 


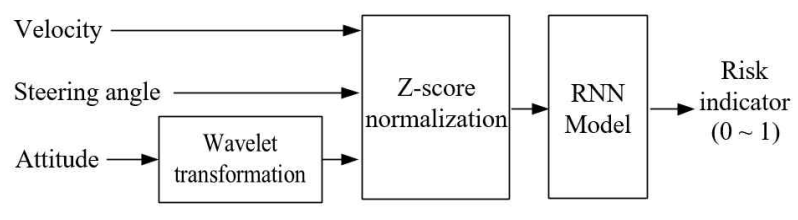

Fig. 11. Risk assessment block diagram.

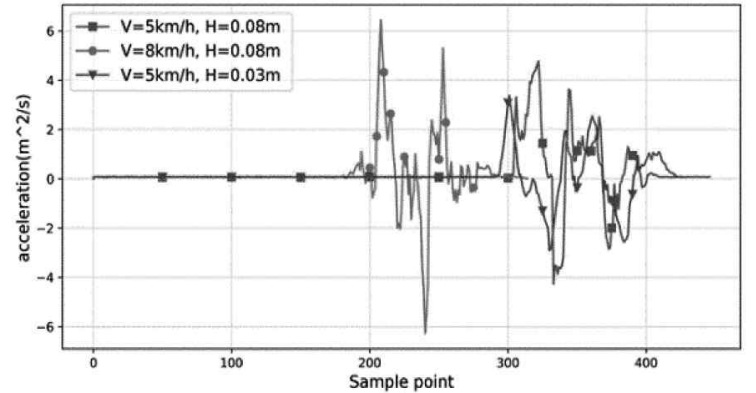

(a)

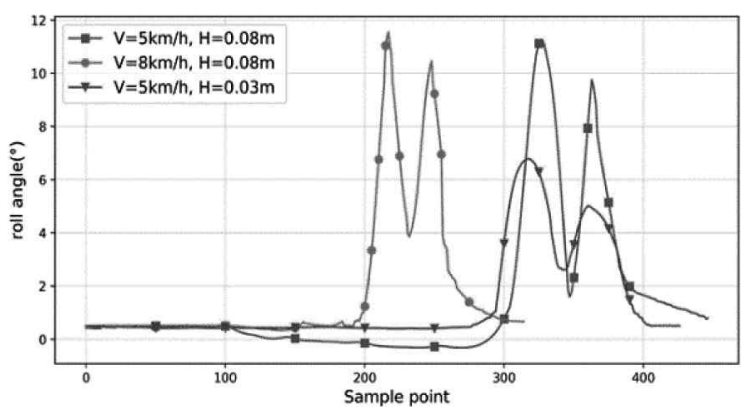

(b)

Fig. 12. The major input of the vehicle crossing over obstacles.

the risk of vehicle rollover is higher.

To verify the validity of the model, we controlled the prototype vehicle to do two operations: Indoor crossing over obstacles operation and outdoor turning operation. The collected data is sent to the trained RNN model and obtain its output.

\subsubsection{Crossing over obstacles}

An experiment with flat surface in the indoor environment was carried out to eliminate the interference from uneven surface when crossing over obstacles. The prototype vehicle was controlled to travel straight (the steering angle is $0^{\circ}$ ) and cross over the obstacle with heights of $0.08 \mathrm{~m}$ and $0.03 \mathrm{~m}$. For our home-built prototype vehicle, it is dangerous to cross the $0.08 \mathrm{~m}$ high obstacle and safe to cross the $0.03 \mathrm{~m}$ high obstacle. The major input and the model output are shown in Figs. 12 and 13.

Fig. 14 shows that the front and rear wheels crossed over the obstacle, respectively, which caused sudden model output changes. More specifically, Fig. 14(a) indicates that the front wheel is on the obstacle and Fig. 14(b) indicates that the rear wheel is over the obstacle. When the vehicle crossed over the $0.03 \mathrm{~m}$ high obstacle at $5 \mathrm{~km} / \mathrm{h}$, as can be seen in Fig. 14(c), the model output did not increase suddenly because the 0.03

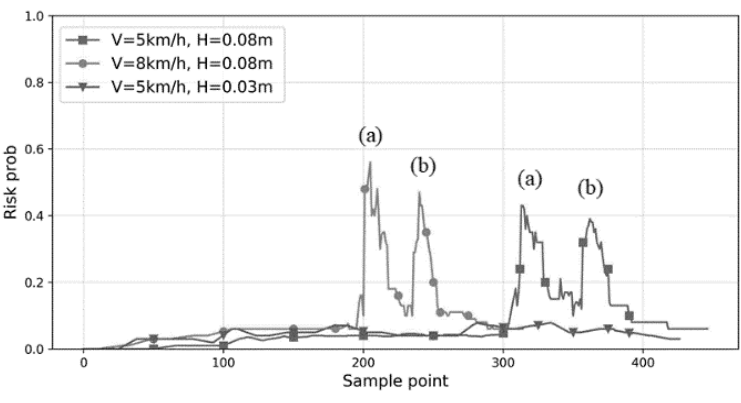

Fig. 13. The model output of crossing over obstacles.

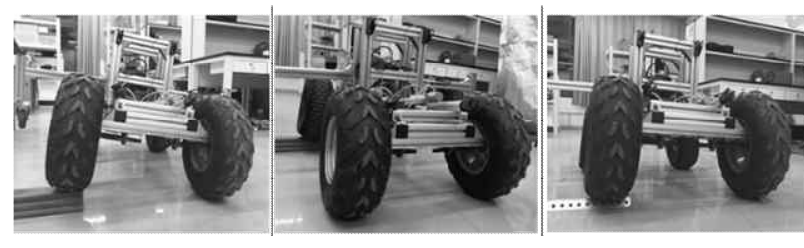

(a)

(b)

(c)

Fig. 14. The prototype vehicle crossing over obstacles: (a) Front wheel crossing over the obstacle with a height of $0.08 \mathrm{~m}$; (b) rear wheel crossing over the obstacle with a height of $0.08 \mathrm{~m}$; (c) crossing over the obstacle with a height of $0.03 \mathrm{~m}$.

$\mathrm{m}$ high obstacle is easy to cross and it has less influence on the roll angle and the lateral acceleration.

When the rear wheel of prototype crossed over the obstacle, the influence on the inclination of the vehicle was smaller than the front wheel cross over it because of the swing rear driving axle mounted on the rear wheel. The peaks of lateral acceleration and roll angle were greatest when the vehicle crossed over the $0.08 \mathrm{~m}$ high obstacle at $8 \mathrm{~km} / \mathrm{h}$ and the output was also greatest. In general, the model reflects the lateral stability of prototype accurately when it crosses over obstacles.

\subsubsection{Turning}

An outdoor environment with an uneven surface, which is similar to the working environment of a real articulated steering vehicle, was used to verify the validity of the model. First, the prototype vehicle did circular motion at $35^{\circ}$ (maximum steering angle) and its velocity increased until a dangerous state occurred. Figs. 15 and 16 show the experimental results. The solid line in Fig. 16 represents the output of the model, and the dotted line represents the actual state of the vehicle ( 0 safe, 1 dangerous).

It can be seen that the vehicle state changed from safety to danger as its speed increased. The roll angle and the lateral acceleration increased at the 3890th sampling point, at which point the vehicle appeared to be dangerous (one-side wheel leaving the ground). The model output began to rise at the 3687th sampling point and reach 0.51 at the 3890th sampling point. As the speed increased further, the vehicle was completely unstable and the output of the model kept above 0.5 , which means the vehicle was always in a dangerous state. The results show a good agreement between the output and the 
Table 4. The average output of circular motion at different steering angles and velocities.

\begin{tabular}{|c|c|c|c|c|c|c|c|c|c|}
\hline \multirow{2}{*}{$\begin{array}{l}\text { Steering } \\
\text { angle }\left({ }^{\circ}\right)\end{array}$} & \multicolumn{9}{|c|}{ Velocity (km /h) } \\
\hline & 4 & 5 & 6 & 7 & 8 & 9 & 10 & 11 & 12 \\
\hline 20 & 0.01 & 0.01 & 0.02 & 0.04 & 0.26 & 0.32 & 0.35 & 0.45 & 0.87 \\
\hline 25 & 0.01 & 0.03 & 0.01 & 0.05 & 0.20 & 0.32 & 0.61 & 0.83 & 0.95 \\
\hline 30 & 0.01 & 0.01 & 0.02 & 0.07 & 0.24 & 0.63 & 0.68 & 0.82 & 0.94 \\
\hline 35 & 0.00 & 0.01 & 0.03 & 0.02 & 0.18 & 0.51 & 0.62 & 0.89 & 0.82 \\
\hline
\end{tabular}

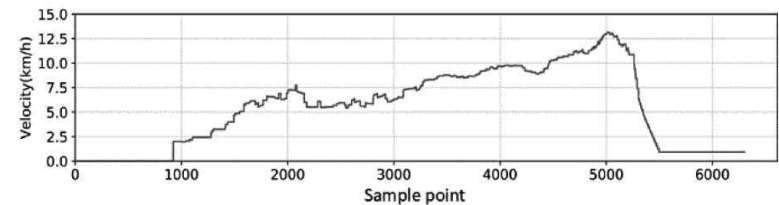

(a)

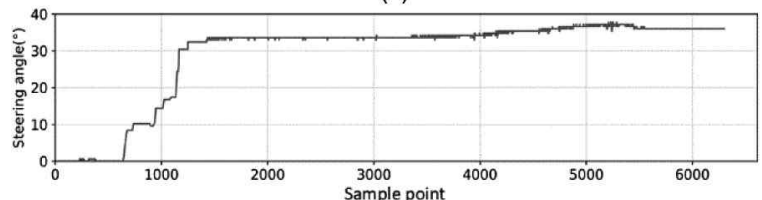

(b)

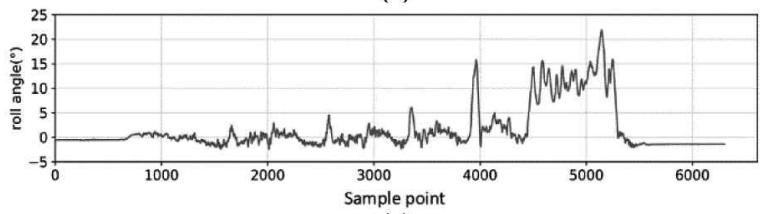

(c)

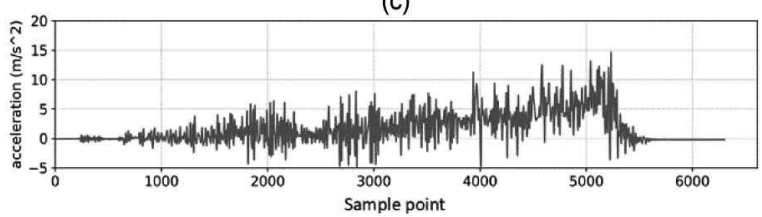

(d)

Fig. 15. The major input of constant velocity circular motion experiment: (a) Velocity; (b) steering angle; (c) roll angle; (d) lateral acceleration after processed.

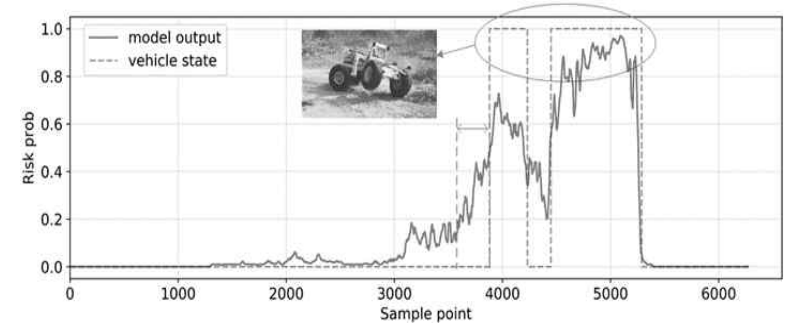

Fig. 16. The model output of constant velocity circular motion experiment.

input data.

Then, we did a circular motion at different steering angles and recorded the average output of the model in typical values of steering angle and velocity. Table 4 summarizes the average output of circular motion at typical steering angles and velocities. The numbers with bold font indicate that the prototype vehicle was in a dangerous state at this time. When the steering angle was $20^{\circ}$, the velocity of the prototype vehicle entering a dangerous state was about $11 \mathrm{~km} / \mathrm{h}$. When the steering angle was $25^{\circ}$, the velocity of the prototype vehicle entering a dangerous state was about $10 \mathrm{~km} / \mathrm{h}$. When the steering angle was $30^{\circ}$ and $35^{\circ}$, the velocity of the prototype vehicle entering a dangerous state was $9 \mathrm{~km} / \mathrm{h}$. However, the output was less than 0.5 . This is the only point the model identified the vehicle state wrongly in the typical values we listed. In general, the output of our RNN model will increase as the steering angle and speed increase and the output accurately reflects the rollover risk when turning.

\section{Conclusions}

This paper has investigated how to effectively assess the rollover risk of articulated steering vehicles. A novel data-driven modelling methodology has been proposed, which needn't build a high dimensional mechanical model for articulated steering vehicles. It is based on reliable data obtained from the most prone rollover condition. much time needs to be spent on data collection. The wavelet transformation was used to process the key data and select RNN model to learn the data we collected. The proposed modelling method was compared with two common data modelling methods. Experiments were conducted on a home-built wheel loader prototype that is 1:4 scale down version of a real XG958 wheel loader. The experimental results demonstrated that the RNN model performs better than SVC and GBDT in test sets and achieves convincing results for rollover risk assessment in turning and crossing obstacles working conditions.

However, the predictability of the model is not perfect due to the lack of terrain features of the road ahead. Extracting the terrain features and feeding it to the model is a main focus of future work. Moreover, it is necessary to transplant the algorithm into a real wheel loader vehicle for test and implementation.

\section{Acknowledgments}

This work was financially supported by the National Natural Science Foundation of China (Grant No. 51575463 and Grand No. 51975495), as well as was supported by Fujian Collaboration Innovation Centre for R\&D of Coach and Special Vehicle (Grant No. 2016BJC016), Major science and technology projects of Xiamen of China (3502Z20191019).

\section{References}

[1] N. L. Azad, A. Khajepour and J. McPhee, A survey of stability enhancement strategies for articulated steer vehicles, Int. J. of Heavy Vehicle Systems, 16 (1) (2009) 26-48.

[2] L. Li, Y. Lu, R. Wang and J. Chen, A three-dimensional dynamics control framework of vehicle lateral stability and rollover prevention via active braking with MPC, IEEE Transactions on Industrial Electronics, 64 (4) (2017) 3389-3401.

[3] F. Yakub, S. Lee and Y. Mori, Comparative study of MPC and LQC with disturbance rejection control for heavy vehicle roll- 
over prevention in an inclement environment, J. of Mechanical Science and Technology, 30 (8) (2016) 3835-3845.

[4] J. Yoon, D. Kim and K. Yi, Design of a rollover index-based vehicle stability control scheme, Vehicle System Dynamics, 45 (5) (2007) 459-475.

[5] X. Li, G. Wang, Z. Yao and J. Qu, Dynamic model and validation of an articulated steering wheel loader on slopes and over obstacles, Vehicle System Dynamics, 51 (9) (2013) 1305-1323.

[6] C. Y. Chi, S. C. Qin, D. W. Yu and W. J. Zhang, The application of pseudo-excitation method to the ride comfort research of wheel-loader, Applied Mechanics and Materials, 34-35 (2010) 538-543.

[7] Y. Yavin, Modelling the motion of an underground mining vehicle, Mathematical and Computer Modelling, 42 (9-10) (2005) 1123-1130.

[8] M. lida, H. Nakashima, H. Tomiyama, T. Oh and T. Nakamura, Small-radius turning performance of an articulated vehicle by direct yaw moment control, Computers and Electronics in Agriculture, 76 (2) (2011) 277-283.

[9] N. Lashgarian Azad, A. Khajepour and J. McPhee, Robust state feedback stabilization of articulated steer vehicles, Vehicle System Dynamics, 45 (3) (2007) 249-275.

[10] T. G. Thuruthel, B. Shih, C. Laschi and M. T. Tolley, Soft robot perception using embedded soft sensors and recurrent neural networks, Science Robotics, 4 (26) (2019) eaav1488.

[11] N. Munir et al., Investigation of deep neural network with drop out for ultrasonic flaw classification in weldments, J. of Mechanical Science and Technology, 32 (7) (2018) 3073-3080.

[12] K. Yeo and I. Melnyk, Deep learning algorithm for data-driven simulation of noisy dynamical system, $\mathrm{J}$ of Computational Physics, 376 (2019) 1212-1231.

[13] Y. Sellami, H. Imine, A. Boubezoul and J.-C. Cadiou, Rollover risk prediction of heavy vehicles by reliability index and empirical modelling, Vehicle System Dynamics, 56 (3) (2017) 385-405.

[14] U. Fugiglando et al., Driving behavior analysis through CAN bus data in an uncontrolled environment, IEEE Transactions on Intelligent Transportation Systems, 20 (2) (2019) 737-748.

[15] H. Liu, T. Taniguchi, Y. Tanaka, K. Takenaka and T. Bando, Visualization of driving behavior based on hidden feature extraction by using deep learning, IEEE Transactions on Intelligent Transportation Systems, 18 (9) (2017) 2477-2489.

[16] N. A. Spielberg, M. Brown, N. R. Kapania, J. C. Kegelman and J. C. Gerdes, Neural network vehicle models for highperformance automated driving, Science Robotics, 4 (28) (2019) eaaw1975.

[17] D. Yang, L. Zhu, Y. Liu, D. Wu and B. Ran, A novel carfollowing control model combining machine learning and kinematics models for automated vehicles, IEEE Transactions on Intelligent Transportation Systems, 20 (6) (2019) 1991-2000.

[18] R. Zhao, R. Yan, Z. Chen, K. Mao, P. Wang and R. X. Gao, Deep learning and its applications to machine health monitoring, Mechanical Systems and Signal Processing, 115 (2019) 213-237.

[19] A. Rahman, V. Srikumar and A. D. Smith, Predicting electricity consumption for commercial and residential buildings using deep recurrent neural networks, Applied Energy, 212 (2018) 372-385.

[20] M. Morchid, Parsimonious memory unit for recurrent neural networks with application to natural language processing, $\mathrm{Neu}$ rocomputing, 314 (2018) 48-64.

[21] M. H. Woo, S. Lee and H. M. Cha, A study on the optimal route design considering time of mobile robot using recurrent neural network and reinforcement learning, J. of Mechanical Science and Technology, 32 (10) (2018) 4933-4939.

[22] Q. Zhu, C. P. Yang, H. Hu and X. F. Wu, Building a novel dynamics rollover model for critical instability state analysis of articulated multibody vehicles, International J of Heavy Vehicle Systems, 1 (2019).

[23] P. Doostdar and J. Keighobadi, Design and implementation of SMO for a nonlinear MIMO AHRS, Mechanical Systems and Signal Processing, 32 (2012) 94-115.

[24] J. Keighobadi, M. Hosseini-Pishrobat, J. Faraji and M. Naseri Langehbiz, Design and experimental evaluation of immersion and invariance observer for low-cost attitude-heading reference system, IEEE Transactions on Industrial Electronics (2019).

[25] G. Phanomchoeng and R. Rajamani, New rollover index for the detection of tripped and untripped rollovers, IEEE Transactions on Industrial Electronics, 60 (10) (2013) 4726-4736.

[26] X. Li, Y. Wu, W. Zhou and Z. Yao, Study on roll instability mechanism and stability index of articulated steering vehicles, Mathematical Problems in Engineering, 2016 (2016) 1-15.

[27] Q. Zhu, J. Yi, H. Chen, C. Wen and H. Hu, Lateral stability simulation and analysis for wheel loaders based on the steadystate margin angle, International J. of Modelling, Identification and Control, 22 (3) (2014) 185-194.

[28] S. G. Mallat, A theory for multiresolution signal decomposition: The wavelet representation, IEEE Transactions on Pattern Analysis and Machine Intelligence, 11 (7) (1989) 674-693.

[29] P. Doostdar, J. Keighobadi and M. A. Hamed, INS/GNSS integration using recurrent fuzzy wavelet neural networks, GPS Solutions, 24 (29) (2020).

[30] J. Keighobadi, K. A. Fazeli and M. S. Shahidi, Selfconstructing neural network modeling and control of an AGV, Positioning, 4 (2) (2013) 160-168.

[31] K. Greff, R. K. Srivastava, J. Koutnik, B. R. Steunebrink and J. Schmidhuber, LSTM: A search space odyssey, IEEE Transactions On Neural Networks And Learning Systems, 28 (10) (2017) 2222-2232.

[32] $\mathrm{X}$. Wu et al., Top 10 algorithms in data mining, Knowledge and Information Systems, 14 (1) (2007) 1-37. 